\title{
RESEARCH HIGHLIGHT OPEN Targeting multiple epitopes on the spike protein: a new hope for COVID-19 antibody therapy
}

\author{
Yeping Sun ${ }^{1}$, Bostjan Kobe $\mathbb{i D}^{2}$ and Jianxun $\mathrm{Qi}^{1,3}$ \\ Signal Transduction and Targeted Therapy (2020)5:208
}

; https://doi.org/10.1038/s41392-020-00320-6
Lihong Liu et al. ${ }^{1}$ from Columbia University recently reported in Nature the isolation of a collection of 19 SARS-CoV-2-neutralizing monoclonal antibodies (mAbs) from five infected patients with high plasma virus-neutralizing titers. These antibodies can neutralize SARS-CoV-2 in vitro, and nine of them exhibited exquisite potency, with $50 \%$ virus-inhibitory concentrations of $9 \mathrm{ng} / \mathrm{mL}$ or less. The strategy for isolation of these antibodies included sorting SARS-CoV-2 spike protein (S)-specific memory B cells by flow cytometry and single-cell sequencing.

Currently, the global effect of the SARS-CoV-2-caused COVID-19 has continued to escalate. The disastrous pandemic has brought to the forefront the urgency and necessity for rapid development of countermeasures. Among the potential countermeasures, one promising candidate is recombinant neutralizing antibodies.

Liu et al. further mapped the epitope locations of these antibodies on the SARS-CoV-2 S by the ELISA assay. They found that some $S$ trimer-binding antibodies bind to the receptorbinding domain (RBD) while the others bind to the $\mathrm{N}$-terminal domain (NTD). A so-called "checkerboard" experiment for competition among antibodies for binding to the $S$ trimer by ELISA grouped the non-RBD antibodies (antibodies that do not bind RBD) into four clusters (A, B, C, and D). Clusters $A, B$, and $C$ are mapped to regions within or near the NTD, and the regions $A$ and $B$ are partially overlapping. The potent neutralizing $m A b s$ fall exclusively into cluster $A$ and bind to a patch on the NTD, while weaker neutralizing $m A b s$ recognize a region at the interface between clusters $A$ and $B$.

The same "checkerboard" experiment identified another four clusters among the RBD-directed mAbs (Clusters E, F, G, and H). Cluster $E$ is a large cluster containing $m A$ bs capable of blocking the receptor ACE2 binding; $m A B s$ of cluster $G$ bind to an epitope on a cryptic site on the side of RBD with an "up" conformation. The most potent neutralizing mAbs group together within cluster $E$, suggesting that they recognize the top of RBD and neutralize the virus by competitive inhibition of receptor binding. Cluster $F$ is likely situated between the S top and the "cryptic" site.

The authors further solved single-particle cryo-EM (electron microscopy) structures of the $S$ trimer in complex with several mAbs binding to different epitopes on the S protein (Fig. 1). The first corresponds to the RBD-directed $m A b$ 2-4, which belongs to cluster $E$ and can block $A C E 2$ binding. The $3.2-\AA$ resolution structures show three Fab fragments of the 2-4-mAb bind to the $S$ trimer. Fab 2-4 binds to the spike protein near the apex and locks the RBDs in a "down" conformation, while also occluding access to ACE2. The second corresponds to the 4-8 Fab (NTD-directed, cluster A). 4-8 Fab binds to the tip of the NTDs, showing two main conformations: one for a three 4-8 Fab-bound complex of S trimer with all the RBDs in the "down" conformation ( $3.9 \AA$ resolution structure), and the other for a three 4-8 Fab-bound complex of $S$ trimer with one RBD in the "up" conformation $(4.0 \AA$ resolution structure). The third corresponds to the $S$ trimer in complex with three 2-43 Fabs, each targeting a quaternary epitope (spanning more than one domain) on the top of RBD (5.8 $\AA$ resolution structure).

Isolation and identification of SARS-CoV-2 S-specific mAbs is now a blooming area. S-specific mAbs are being continually reported by different researchers. For example, Lv et al. reported the potent neutralizing mAb H014, which targets the side of the $\mathrm{RBD}$ in the open (up) conformation. ${ }^{2}$ Shi et al. $^{3}$ reported that the $\mathrm{mAb}$ CB6 neutralizes SARS-CoV-2 by recognizing an epitope overlapping with the ACE2-binding site. Wu et al. ${ }^{4}$ reported a noncompeting mAb pair, B38 and H4, which block RBD-ACE2 binding by targeting different epitopes on RBD. A startling highlight of Liu et al.'s report is that they successfully identified a collection of many mAbs that target multiple epitopes. Among their most potent $9 \mathrm{mAbs}, 4$ are directed to the RBD, 3 directed to the NTD, and 2 to quaternary epitopes. It is important that there are epitopes on the NTD that can be neutralizing, although the mechanisms for neutralization are unknown.

The variety of the potent neutralizing mAbs against SARS-CoV2 reported by Liu et al. and other researchers inspires optimism that we will be able to find the highly effective and safe candidates for clinical treatment of the COVID-19. Most importantly, they provide opportunities for development of effective mAb cocktail treatments. As shown by Baum et al. ${ }^{5}$, the noncompeting antibody cocktail was not able to induce SARS-CoV-2 S escape mutations. Therefore, the mAbs targeting multiple nonoverlapping epitopes, including those discovered by Liu et al., pave a way toward developing promising $\mathrm{mAb}$ cocktail therapies for COVID-19.

\footnotetext{
${ }^{1}$ CAS Key Laboratory of Pathogenic Microbiology and Immunology, Institute of Microbiology, Chinese Academy of Sciences, 100101 Beijing, China; ${ }^{2}$ School of Chemistry and

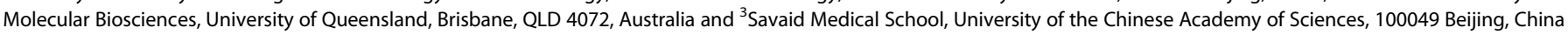
Correspondence: Bostjan Kobe (b.kobe@uq.edu.au) or Jianxun Qi (jxqi@im.ac.cn)
}

Received: 10 August 2020 Revised: 25 August 2020 Accepted: 2 September 2020

Published online: 19 September 2020 
a

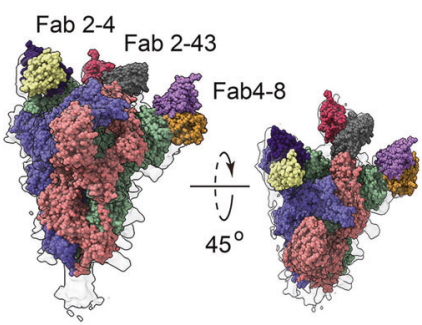

C

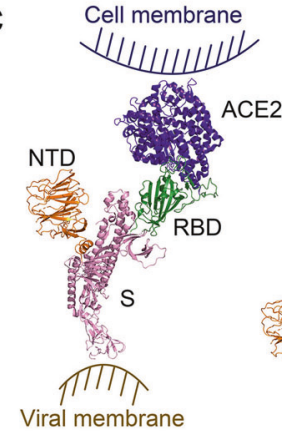

b

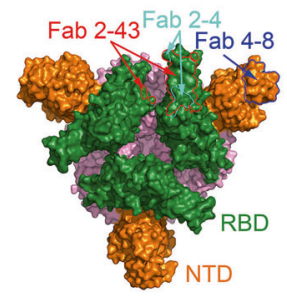

d<smiles>C#CC#CC</smiles>

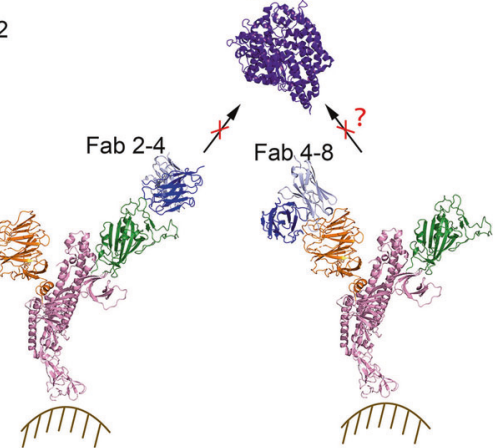

Fig. 1 Neutralizing mAbs targeting different epitopes on SARS-CoV$2 \mathrm{~S}$ inhibit virus infection. a A structural model for SARS-CoV-2 S in complex mAbs Fab 2-4, Fab 4-8, and Fab 2-43. b Target regions of Fab 2-4, Fab 4-8, and Fab 2-43. c SARS-CoV-2 S (for clarification, only one $\mathbf{S}$ monomer is shown) binds to ACE2 receptor. $\mathbf{d}$ The RBDdirected Fab 2-4 inhibits virus infection via blocking SARS-CoV-2 S binding to ACE2 receptor; the NTD-directed Fab 4-8 inhibits virus infection via unknown mechanisms

\section{ACKNOWLEDGEMENTS}

This work was supported by the Strategic Priority Research Program of CAS (XDB37030204), the National Key Research and Development Program of China (2016YFD0500305), and the Zhejiang University Special Scientific Research Fund for COVID-19 Prevention and Control (2020XGZX031).

\section{ADDITIONAL INFORMATION}

Competing interests: The authors declare no competing interests.

\section{REFERENCES}

1. Liu, L. et al. Potent neutralizing antibodies directed to multiple epitopes on SARSCoV-2 spike. Nature https://doi.org/10.1038/s41586-020-2571-7 (2020).

2. Lv, Z. et al. Structural basis for neutralization of SARS-CoV-2 and SARS-CoV by a potent therapeutic antibody. Science https://doi.org/10.1126/science.abc5881 (2020).

3. Shi, R. et al. A human neutralizing antibody targets the receptor-binding site of SARS-CoV-2. Nature https://doi.org/10.1038/s41586-020-2381-y (2020).

4. $\mathrm{Wu}$, Y. et al. A noncompeting pair of human neutralizing antibodies block COVID19 virus binding to its receptor ACE2. Science 368, 1274-1278 (2020).

5. Baum, A. et al. Antibody cocktail to SARS-CoV-2 spike protein prevents rapid mutational escape seen with individual antibodies. Science https://doi.org/ 10.1126/science.abd0831 (2020).

(c) Open Access This article is licensed under a Creative Commons BY Attribution 4.0 International License, which permits use, sharing, adaptation, distribution and reproduction in any medium or format, as long as you give appropriate credit to the original author(s) and the source, provide a link to the Creative Commons license, and indicate if changes were made. The images or other third party material in this article are included in the article's Creative Commons license, unless indicated otherwise in a credit line to the material. If material is not included in the article's Creative Commons license and your intended use is not permitted by statutory regulation or exceeds the permitted use, you will need to obtain permission directly from the copyright holder. To view a copy of this license, visit http://creativecommons. org/licenses/by/4.0/.

(c) The Author(s) 2020 